\title{
Nasopharyngeal flora in children with acute otitis media before and after implementation of 7 valent pneumococcal conjugate vaccine in France
}

\author{
Robert Cohen ${ }^{1,2^{*}}$, Edouard Bingen ${ }^{3}$, Corinne Levy ${ }^{1}$, Franck Thollot ${ }^{4}$, Michel Boucherat $^{1}$, Véronique Derkx ${ }^{1}$ and \\ Emmanuelle Varon ${ }^{5}$
}

\begin{abstract}
Background: Several studies have investigated the impact of 7-valent pneumococcal conjugate vaccine (PCV7) on pneumococcal (Sp) and staphylococcal (Sa) nasopharyngeal (NP) carriage. Few have investigated the impact on Haemophilus influenzae (Hi) and Moraxella catarrhalis (Mc) carriage. We aimed to compare the NP carriage rates in young children with acute otitis media (AOM) before and after PCV7 implementation in France.

Methods: Prior to PCV7 implementation, we performed 4 successive randomized trials with NP samples. These studies compared several antibiotic regimens for treating AOM in young children (6 to 30 months). After PCV7 implementation, to assess the impact of the vaccination program on NP flora, young children with AOM were enrolled in a prospective surveillance study. In each study, we obtained an NP sample to analyze the carriage rates of Sp, Hi, Mc and Sa and the factors influencing the carriage. Standardized history and physical examination findings were recorded; the methods used for NP swabs (sampling and cultures) were the same in all studies.

Results: We enrolled 4,405 children (mean age 13.9 months, median 12.8). Among the 2,598 children enrolled after PCV7 implementation, 98.3\% were vaccinated with PCV7. In comparing the pre- and post-PCV7 periods, we found a slight but non-significant decrease in carriage rates of pneumococcus (AOR $=0.85$ [0.69;1.05]), H. influenzae (AOR $=0.89[0.73 ; 1.09]$ ) and S. aureus (AOR $=0.92[0.70 ; 1.19])$. By contrast, the carriage rate of $M$. catarrhalis increased slightly but not significantly between the 2 periods ( $A O R=1.08[0.95 ; 1.2]$ ). Among $S p$ carriers, the proportion of PCV7 vaccine types decreased from $66.6 \%$ to $10.7 \%(P<0.001)$, penicillin intermediate-resistant strains increased from $30.3 \%$ to $43.4 \%(P<0.001)$, and penicillin-resistant strains decreased greatly from $22.8 \%$ to $3.8 \%(P<0.001)$. The proportion of Hi B-lactamase-producing strains decreased from 38.6\% to 17.1\% ( $P<0.001)$.

Conclusion: The carriage rates of otopathogen species (Sp, Hi, Mc) and Sa did not significantly change in children with AOM after PCV7 implementation in France. However, we observed significant changes in carriage rates of PCV7 vaccine serotypes and penicillin non-susceptible Sp.
\end{abstract}

\section{Background}

The nasopharynx is normally colonized with bacteria such as Streptococcus viridans, nonhemolytic streptococci, diphtheroids, and Neisseria sp. and potential middle-ear pathogens such as Streptococcus pneumoniae (Sp), non-typable Haemophilus influenzae (NTHi) and Moraxella catarrhalis (Mc) [1]. Furthermore, this

\footnotetext{
* Correspondence: robert.cohen@wanadoo.fr

'ACTIV, Association Clinique Thérapeutique Infantile du Val de Marne, 27 rue Inkermann, F94100 Saint Maur des Fossés, France

Full list of author information is available at the end of the article
}

ecosystem is the reservoir for Staphylococcus aureus (Sa) strains implicated in several infections [2]. The rates of colonization of these potentially pathogenic species are heterogeneously reported in the literature. In fact, in addition to technical procedures of sampling or culture, many other factors may affect colonization rates: daycare attendance; respiratory illness, including acute otitis media (AOM); symptoms of AOM; crowding; season; siblings; and immunization status [3]. Although Sp, $\mathrm{NTHi}$, and Mc are part of the normal nasopharyngeal (NP) flora, increased rate of colonization may identify a

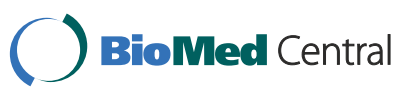

(c) 2012 Cohen et al; licensee BioMed Central Ltd. This is an Open Access article distributed under the terms of the Creative Commons Attribution License (http://creativecommons.org/licenses/by/2.0), which permits unrestricted use, distribution, and reproduction in any medium, provided the original work is properly cited. 
subpopulation of children with increased risk of AOM. Indeed, frequency of colonization and AOM is highly correlated $(r=0.37, \mathrm{P}<.001)$ for each pathogen [4]. Furthermore, the disease potential of these pathogens are not identical. Sp is more frequently associated with severe AOM (high fever, otorrhea, mastoïditis), pneumonia and invasive diseases and NTHi with conjunctivitis and recurrent AOM $[5,6]$. Finally, Mc appears to be the less pathogenic organism [7].

French authorities recommended routine infant immunization with 7 -valent pneumococcal conjugate vaccine (PCV7) at age 2, 4 and 12 months for all children [8]. The national PCV7 vaccination coverage has increased slowly and is now relatively high: at least one dose of PCV7 was received by $69 \%$ of children less than 2 years old in 2007, 85\% in 2008 and $90 \%$ in 2009 as compared with $62 \%$ in 2006 [9].

Before, during, and after PCV7 implementation, several studies investigated the impact of PCV7 on pneumococcal and staphylococcal carriage [10-12], but few have investigated the impact on NTHi and Mc carriage. Subtle modifications in the equilibrium of NP flora could lead to different etiological profiles of AOM and eventually other respiratory tract infections. We aimed to compare NP carriage of Sp, NTHi, Mc and Sa in young children (6 to 30 months) with AOM before and after PCV7 implementation in France.

\section{Methods}

\section{Study design}

Before the PCV7 implementation, we performed 4 randomized trials on antibiotic treatment for AOM. These studies used standardized protocols (including bacteriological NP samples at enrolment), the same inclusion criteria of AOM [13] and the same age of enrolled children [3,14-16]. Furthermore, the studies involved mostly the same investigators and the same centralized microbiology laboratories. These studies allowed us to identify risk factors for carriage of NP flora bacterial species in children with AOM and to build a protocol for monitoring the impact of the introduction of PCV7 on this ecological niche [3,12,17-20]. Therefore, for the pre-PCV7 implementation period (1993-2000), we used the dataset from the 4 randomized trials [3,14-16], and for the postPCV7 implementation period (2006- 2009), we used data from an ongoing surveillance study on the impact of PCV7 on NP carriage [12,17-20]. To take into account herd immunity, for the post-PCV7 period, all children were enrolled regardless of their PCV7 vaccination status.

The protocols were approved by the Saint Germain en Laye Hospital Ethics Committee, and written informed consent was obtained from the parents or guardians.
The inclusion criteria and definitions were the same during the two periods.

The study populations consisted of children of both sexes; age 4 to 30 months, with newly diagnosed AOM, recruited by pediatricians in pediatric outpatient clinics throughout France. Diagnostic criteria for AOM included the Paradise algorithm for acute suppurative otitis media (effusion + marked redness or marked bulging or moderate redness and bulging) [13]. Patients were not eligible if they had received antibiotic treatment within the 7 days before enrolment. Standardized history and physical examination findings were recorded, and information gathered at study entry included sex, age, daycare modalities, recent antibiotic treatment (within 3 months before enrolment), history of AOM, and clinical symptoms and signs of AOM (thoroughly documented in an identical manner in all trials). The types of antibiotic treatment used by the patients in the studies were most commonly amoxicillinclavulanate and cefpodoxime proxetil.

Daycare modalities were defined according to those most frequently used in France:- Children cared for at home by parents or another person only in the presence of children belonging to the family.- Children cared for by a childcare giver, outside the home, in the presence of other children; a maximum of 3 children is authorized.- Children cared for in a daycare center, which can include many children. In general, one room should be used for the care of no more than 10 children of the same age.

Upon inclusion, the patients were sampled in order to identify bacterial pathogens colonizing the NP niche.

\section{Microbiologic investigations}

The same methods for NP sampling and microbiological testing were used in all studies. Deep NP samples were taken transnasally with use of a flexible, sterile, soft rayon swab tip. After sampling, swabs were immediately inoculated in transport medium (Copan Venturi Transystem, Brescia, Italy), stored at room temperature and sent within $48 \mathrm{hr}$ to laboratories at the National Reference Center for Pneumococci at G. Pompidou European Hospital and to Robert Debré Hospital, in Paris. Swabs were plated within $24 \mathrm{hr}$ onto chocolate agar, S. aureus ID agar (SAID; bioMérieux, La Balme Les Grottes, France) $5 \%$ horse blood agar. Agar plates were incubated at $37^{\circ} \mathrm{C}$ for $48 \mathrm{hr}$ under $5 \% \mathrm{CO}_{2}$ for chocolate and blood agar. Isolates of $\mathrm{Sp}, \mathrm{Hi}, \mathrm{Mc}$ and $\mathrm{Sa}$ were identified using colony morphology and conventional methods of determination. S. pneumoniae serotyping and antibiotic susceptibility testing were performed at the National Reference Center for Pneumococci. Serotyping was performed using latex particles, which were sensitized with 
antisera purchased from the Statens Serum Institut (Copenhagen, Denmark). Susceptibility of S. pneumoniae isolates to penicillin $\mathrm{G}$ was determined from minimal inhibitory concentrations (MICs) by the agar-dilution method. Isolates were classified as penicillin susceptible ( $\mathrm{MIC} \leq 0.06 \mu \mathrm{g} / \mathrm{ml})$, penicillin non-susceptible (MIC > $0.12 \mu \mathrm{g} / \mathrm{ml})$, penicillin intermediate resistant $(0.12 \leq$ MIC $\leq 1.0 \mu \mathrm{g} / \mathrm{ml}$ ), or penicillin resistant (MIC $\geq 2 \mu \mathrm{g} /$ $\mathrm{ml}$ ) according to the Clinical and Laboratory Standards Institute [21]. H. influenzae isolates underwent capsular serotyping by the slide agglutination method with specific antisera (Phadebact, Boule Diagnostic, Huddinge, Sweden). The production of $B$-lactamase was assessed by a chromogenic cephalosporin test (Nitrocefin; Cefinase; Biomerieux, Marcy l'Etoile, France). H. influenzae strains were further classified as ampicillin susceptible $(\mathrm{MIC} \leq 1 \mathrm{mg} / \mathrm{L})$ or resistant $(\mathrm{MIC}>1 \mathrm{mg} / \mathrm{L})$.

\section{Statistical analyses}

Data were double-entered using 4D software (version 6.4), and analysed using Stata SE 9.1 (Stata Corp., College Station, TX, USA) for univariate analysis and multivariate logistic regression (adjusted odds ratios [AORs] and $95 \%$ confidence intervals [CI]). For the descriptive statistics, we used mean and median age (months) of children. The Pearson chi-square test was used to compare NP carriage of pneumococci, Hi, Mc and Sa before and after PCV7 implementation. Factors related to NP carriage were identified by univariate analysis $(\mathrm{p}<0.20$, Pearson chi-square test). These variables, included in multivariate logistic regression models were daycare attendance, recent antibiotic treatment (within 3 months before enrolment), fever $\geq 38.5^{\circ} \mathrm{C}$, conjunctivitis, otalgia, and carriage of pneumococci, $H$. influenzae, M. catarrhalis and S. aureus. These models were systematically adjusted on the period of study (before and after PCV7 implementation) and on age dichotomized as $<12$ and $\geq 12$ months. The cut-off of 12 months was chosen for regression model for several reasons: the vaccination schedules differ before and after this age (reflecting the immunity maturation), in many studies, the NP carriage is higher after 12 months, and in one of our studies, young age $(<12$ months) predicted penicillin non susceptible pneumococci carriage [19].

\section{Results}

\section{Study population}

The total study population consisted of 4,405 children (mean age 13.9 months, median 12.8): 1,807 were enrolled before PCV7 implementation and 2,598 after. Among the latter group, 98.3\% were PCV7 vaccinated. Less than $1 \%$ of children had received only one dose of PCV7, and $98.6 \%$ were correctly vaccinated for age. Table 1 describes the demographic and clinical characteristics of the 2 populations. Fever $\left(>38.5^{\circ} \mathrm{C}\right)$ and daycare center attendance were more frequent after than before PCV7 implementation. The mean and the median age were slightly higher in the pre-PCV7 period, however, these differences did not exceed 1 month. Otalgia and antibiotic use before enrolment were significantly more frequent before than after PCV7 implementation, with no significant change in type of antibiotic prescribed 3 months before enrolment.

\section{NP colonization in the pre-and post-PCV7 periods}

Table 2 presents the carriage rates of $\mathrm{Sp}, \mathrm{Hi}, \mathrm{Mc}$ and Sa before and after PCV7 implementation. Almost all Hi strains were non-typeable (98.5\%). The carriage rates of Sp, NTHi and Sa did not differ between the 2 periods, but Mc carriage increased (5\%). The Figure 1

Table 1 Demographic and clinical characteristics of children with acute otitis media (AOM) before and after 7-valent pneumococcal conjugate vaccine (PCV7) implementation in France

\begin{tabular}{llll}
\hline Child characteristics & Before PCV7 $\mathbf{n}=\mathbf{1 . 8 0 7}(\%)$ & After PCV7 $\mathbf{n}=\mathbf{2 , 5 9 8}$ (\%) & - value \\
\hline Male sex' & $970(53.7)$ & $1,376(53.0)$ & 0.6 \\
\hline Age (months), mean \pm SD & $14.6 \pm 7.6$ & $13.8 \pm 5.1$ & $<0.0001$ \\
\hline Median & 12.8 & 13.1 & $<0.0001$ \\
\hline Type of care & & $1032(39.7)$ & $<11(31.2)$ \\
\hline Daycare center & $436(24.1)$ & $754(29.1)$ & $<0.0001$ \\
\hline Child caregiver & $530(29.3)$ & $1243(47.9)$ & 0.5 \\
\hline Home & $841(46.6)$ & $652(25.1)$ & $<0.0001$ \\
\hline Antibiotics 3 months before enrolment & $336(57.2)^{*}$ & $1945(75.0)$ & $<0.0001$ \\
\hline Conjunctivitis & $436(24.1)$ & $1551(60.3)$ & \\
\hline Otalgia & $1304(83.0)^{* *}$ & & \\
\hline
\end{tabular}

* available for 587 patients

** available for 1,571 patients 
Table 2 Nasopharyngeal carriage of Streptococcus pneumoniae, Haemophilus influenzae, Moraxella catarrhalis and Staphylococcus aureus in children with AOM before and after PCV7 implementation

\begin{tabular}{llll}
\hline Carriage & Before PCV7 $\mathbf{n}=\mathbf{1 8 0 7}(\%)$ & After PCV7 $\mathbf{n} \mathbf{2 5 9 8}$ (\%) & p-value* \\
\hline S. pneumoniae & $1047(57.9)$ & $1510(58.1)$ & 0.9 \\
\hline PCV7 vaccine types & $402 / 604^{* *}(66.6)$ & $162(10.7)$ & 0.0001 \\
\hline Penicillin intermediate- resistant & $317(30.3)$ & $656(43.4)$ & 0.0001 \\
\hline Penicillin resistant & $239(22.8)$ & $58(3.8)$ & 0.0001 \\
\hline Non-typable H. influenzae & $849(47)$ & $1269(48.8)$ & 0.2 \\
\hline B-lactamase+ & $328(38.6)$ & $217(17.1)$ & 0.0001 \\
\hline M. catarrhalis & $939(52)$ & $1479(56.9)$ & 0.001 \\
\hline S. aureus & $112(6.2)$ & $136(5.2)$ & 0.2 \\
\hline Multiple carriage $(\geq 2$ species) & $978(54.1)$ & $1502(57.8)$ & 0.02 \\
\hline No carriage & $133(7.4)$ & $187(7.2)$ & 0.8
\end{tabular}

* Chi square test

** on available serotypes

presents the distribution of serotypes before and after PCV7 implementation. Among the Sp carriers, the proportion of $\mathrm{PCV} 7$ vaccine types (serotypes $4,6 \mathrm{~B}, 9 \mathrm{~V}$, $14,18 \mathrm{C}, 19 \mathrm{~F}$ and $23 \mathrm{~F}$ ) decreased from $66.6 \%$ to $10.7 \%(\mathrm{P}<0.001)$, penicillin intermediate-resistant strains increased from $30.3 \%$ to $43.4 \%$ ( $\mathrm{P}<0.001)$, and penicillin-resistant strains greatly decreased from $22.8 \%$ to $3.8 \%(\mathrm{P}<0.001)$. The proportion of $\mathrm{Hi} ß-$ lactamase-producing strains decreased from $38.6 \%$ to $17.1 \%(\mathrm{P}<0.001)$.

Risk factors for carriage of S. pneumoniae, NT H.

influenzae, M. catarrhalis, and S. aureus

We analyzed the factors influencing Sp, NTHI, Mc and Sa carriage by logistic regression analysis (Table 3) and found the following:

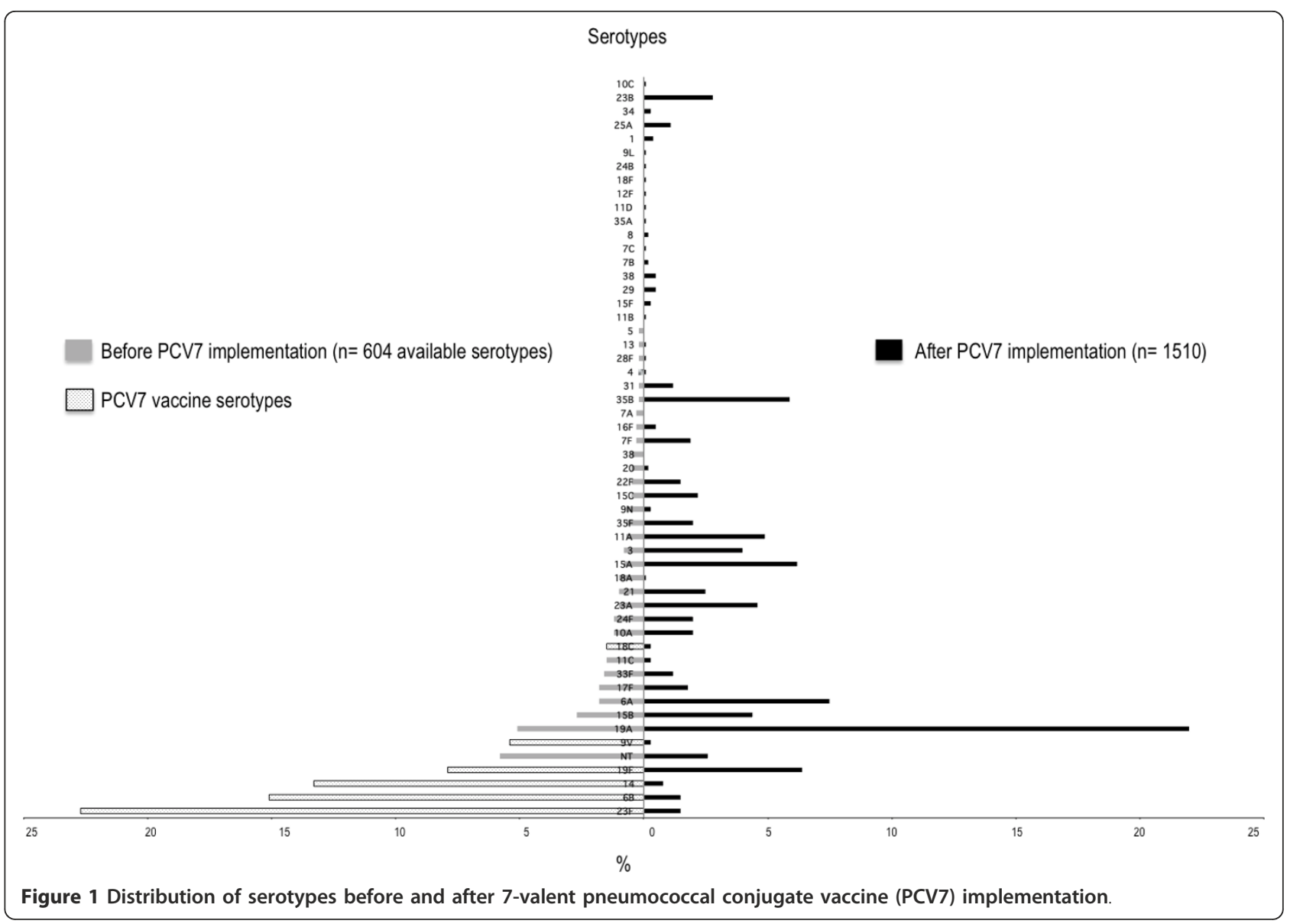


Table 3 Risk factors for carriage of S. pneumoniae, non-typable (NT) H. influenzae, M. catarrhalis, and S. aureus by univariate and multivariate analysis

\begin{tabular}{|c|c|c|c|c|}
\hline & \multicolumn{2}{|c|}{ Univariate analysis } & \multicolumn{2}{|c|}{ Multivariate analysis* } \\
\hline & OR $[95 \% \mathrm{Cl}]$ & $P$ value & OR $[95 \% \mathrm{Cl}]$ & $P$ value \\
\hline \multicolumn{5}{|l|}{ Carriage of S. pneumoniae } \\
\hline Fever $\geq 38.5^{\circ} \mathrm{C}$ & $1.471 .30 ; 1.66$ & $<0.0001$ & $1.31 .11 ; 1.54$ & 0.002 \\
\hline Daycare centre attendance & $1.451 .28 ; 1.65$ & $<0.0001$ & $1.511 .28 ; 1.78$ & $<0.0001$ \\
\hline Carriage of $M$. catarrhalis & $1.411 .25 ; 1.59$ & $<0.0001$ & $1.171 .01 ; 1.36$ & 0.034 \\
\hline Period of the study & $1.010 .89 ; 1.14$ & 0.905 & $0.850 .69 ; 1.05$ & 0.130 \\
\hline Age $\geq 12$ months & $0.990 .88 ; 1.11$ & 0.853 & $0.940 .81 ; 1.09$ & 0.392 \\
\hline Antibiotic use 3 months before enrolment & $0.800 .69 ; 0.92$ & 0.002 & $0.760 .66 ; 0.89$ & $<0.0001$ \\
\hline Carriage of $\mathrm{H}$. influenzae & $0.800 .71 ; 0.90$ & $<0.0001$ & $0.840 .72 ; 0.98$ & 0.028 \\
\hline Conjunctivitis & $0.50 \quad 0.43 ; 0.57$ & $<0.0001$ & $0.520 .44 ; 0.62$ & $<0.0001$ \\
\hline Carriage of S. aureus & $0.360 .27 ; 0.46$ & $<0.0001$ & $0.490 .35 ; 0.67$ & $<0.0001$ \\
\hline \multicolumn{5}{|l|}{ Carriage of NT $H$. influenzae } \\
\hline Conjunctivitis & $4.643 .98 ; 5.42$ & $<0.0001$ & $4.393 .67 ; 5.26$ & $<0.0001$ \\
\hline Daycare centre attendance & $1.631 .44 ; 1.85$ & $<0.0001$ & $1.401 .18 ; 1.65$ & $<0.0001$ \\
\hline Age $\geq 12$ months & $1.431 .27 ; 1.62$ & $<0.0001$ & $1.281 .10 ; 1.48$ & 0.001 \\
\hline Antibiotic use 3 months before enrolment & $1.271 .10 ; 1.46$ & 0.001 & $1.221 .05 ; 1.42$ & 0.01 \\
\hline Period of the study & $1.080 .96 ; 1.22$ & 0.224 & $0.890 .73 ; 1.08$ & 0.261 \\
\hline Carriage of S. pneumoniae & $0.800 .71 ; 0.90$ & $<0.0001$ & $0.830 .71 ; 0.96$ & 0.015 \\
\hline Carriage of S. aureus & $0.470 .35 ; 0.62$ & $<0.0001$ & $0.610 .43 ; 0.85$ & 0.004 \\
\hline \multicolumn{5}{|l|}{ Carriage of $M$. catarrhalis } \\
\hline Daycare centre attendance & $1.911 .68 ; 2.17$ & $<0.0001$ & $1.831 .60 ; 2.09$ & $<0.0001$ \\
\hline Carriage of S. pneumoniae & $1.411 .25 ; 1.59$ & $<0.0001$ & $1.231 .08 ; 1.39$ & 0.001 \\
\hline Fever $\geq 38.5^{\circ} \mathrm{C}$ & $1.301 .15 ; 1.46$ & $<0.0001$ & $1.171 .03 ; 1.33$ & 0.015 \\
\hline Period of the study & $1.221 .08 ; 1.38$ & 0.001 & $1.080 .95 ; 1.22$ & 0.255 \\
\hline Age $\geq 12$ months & $1.141 .01 ; 1.28$ & 0.037 & $1.050 .93 ; 1.19$ & 0.424 \\
\hline Conjunctivitis & $0.790 .68 ; 0.90$ & 0.001 & $0.770 .67 ; 0.89$ & $<0.0001$ \\
\hline Carriage of S. aureus & $0.400 .31 ; 0.52$ & $<0.0001$ & $0.440 .33 ; 0.58$ & $<0.0001$ \\
\hline \multicolumn{5}{|l|}{ Carriage of S. aureus } \\
\hline Carriage of S. pneumoniae & $0.360 .27 ; 0.47$ & $<0.0001$ & $0.350 .26 ; 0.46$ & $<0.0001$ \\
\hline Carriage of $\mathrm{H}$. influenzae & $0.470 .35 ; 0.62$ & $<0.0001$ & $0.430 .33 ; 0.57$ & $<0.0001$ \\
\hline Carriage of $M$. catarrhalis & $0.400 .31 ; 0.52$ & $<0.0001$ & $0.420 .32 ; 0.55$ & $<0.0001$ \\
\hline Age $\geq 12$ months & $0.610 .47 ; 0.79$ & $<0.0001$ & $0.650 .50 ; 0.85$ & 0.002 \\
\hline Period of the study & $0.840 .65 ; 1.08$ & 0.173 & $0.920 .70 ; 1.19$ & 0.521 \\
\hline
\end{tabular}

$\mathrm{OR}=$ odds ratio; $95 \% \mathrm{Cl}=95 \%$ confidence interval.

*Tested variables in the regression logistic model were: period of the study, age, daycare attendances modalities, conjunctivitis, fever $\geq 38.5^{\circ} \mathrm{C}$, otalgia, antibiotic 3 months before inclusion, $\mathrm{Sp}, \mathrm{Hi}, \mathrm{Bc}$ and Sa carriage. Only variables kept in the final model are presented in the table.

- Daycare center attendance was associated with increased carriage rates of Sp, NTHi and Mc.

- Age > 12 months was associated with increased NTHi carriage and with decreased Sa carriage.

- Fever $\geq 38.5^{\circ} \mathrm{C}$ was associated with an increased risk of Sp carriage.

- Conjunctivitis was associated with an increased risk of NTHi carriage and a decreased risk of Sp carriage.

Among potential biologic interactions, Sa carriage was associated with a reduction of Sp, NTHi and Mc carriage. By contrast, Mc carriage was associated with an increased risk of Sp carriage (AOR $=1.23,95 \% \mathrm{CI}[1.08 ; 1.39]$ ).

In comparing the pre- and post-PCV7 periods, we found a slight but non-significant decrease in carriage rates of pneumococcus (AOR $=0.85[0.69 ; 1.05]), H$. influenzae $(\mathrm{AOR}=0.89[0.73 ; 1.09])$ and $S$. aureus (AOR $=0.92[0.70 ; 1.19])$. By contrast, the carriage rate of $M$. catarrhalis increased slightly but not significantly between the 2 periods $(\mathrm{AOR}=1.08[0.95 ; 1.2]$ ). 


\section{Discussion}

In this study, we studied NP samples of children with AOM before and after PCV7 implementation in France and found a marked reduction in vaccine serotype carriage after PCV7 implementation.

However, the overall pneumococcal, $H$. influenzae and S. aureus carriage slightly decreased but non significantly. These results agree with those from 2 prospective studies comparing vaccinated and non-vaccinated populations, one enrolling HIV-infected and HIV-uninfected children 5 years after completion of immunization in South Africa [22] and the other a randomized controlled trial of the impact of 2 doses and $2+1$ doses of PCV7 on carriage of Sp, $\mathrm{Hi}$ and Mc in normal children in The Netherlands [22,23]. Reduced-dose PCV7 schedules slightly reduced overall pneumococcal carriage but not carriage of $\mathrm{Hi}$ and Mc $[22,23]$. The vaccination coverage reported in our study (>98\% in 2006-2009) is higher than that for all children in France (91\%) [9]. However, the investigators in this study were welltrained pediatricians, and the vaccination coverage is higher in France for children followed by pediatricians than general practitioners.

Post-licensure studies of middle ear fluid of patients with AOM after PCV7 implementation in the United States have shown a relative increase in $H$. influenzaeand $M$. catarrhalis-positive middle-ear fluid cultures, together with decreased pneumococcal and vaccine serotype AOM. These were ecological and uncontrolled observations and do not necessarily represent true increases in otitis media due to non-pneumococcal pathogens [24]. The reduction of ß-lactamase-producing NTHi strains is probably due to the overall reduction in antibiotic prescriptions in France with the Health Authorities promotional campaign for appropriate antibiotic prescription $[25,26]$. In fact, the most prescribed antibiotics for AOM (leading reason for antibiotic prescriptions for infants and toddlers) were cefpodoxime proxetil and amoxicillin-clavulanate [25]. Under these selective pressures, ß-lactamase-producing NTHi strains have no ecological advantage. Of note, these regimens could lead to increased B-lactamase-negative ampicillinresistance strains in France [27].

Our study has several limitations. The dataset used for the pre-PCV7 period combined the results of 4 different studies performed during 7 years. Because of the relative heterogeneity of the results, several epidemiological and clinical characteristics (daycare attendance, antibiotics, fever or otalgia) that might influence carriage rates significantly differed between the 2 periods, which necessitated multivariate analysis. Also data for some clinical and demographic features were missing in the pre-PCV7 period. Finally, data that may also influence carriage rates, such as crowding or siblings, were not recorded before and after PCV7 implementation.

Recently available pneumococcal conjugate vaccines with broader serotype coverage may lead to reduced overall pneumococcal carriage; the impact on the carriage of other NP pathogens requires on-going monitoring.

\section{Conclusions}

After implementation of PCV7 vaccine in France, no significant changes in NP carriage with S. pneumoniae, $H$. influenzae, $M$. catarrhalis and $S$. aureus were observed in children with AOM. Effects over time with high-vaccine pressure following nationwide implementation of pneumococcal conjugate vaccines with broader serotype coverage need to be followed.

\section{Acknowledgements}

We thank all paediatricians who participated in the study.

We are grateful to Stéphane Béchet, Dr Elvira Martin, Sophie Grondin, Sylvie Simon, Sadia Tortorelli and Nathalie Kohn for technical assistance.

Financial support was given by Pfizer France.

\section{Author details}

'ACTIV, Association Clinique Thérapeutique Infantile du Val de Marne, 27 rue Inkermann, F94100 Saint Maur des Fossés, France. ${ }^{2}$ Department of Microbiology, CHI 40 Avenue de Verdun, Créteil, France. ${ }^{3}$ Department of Microbiology, Université Denis-Diderot-Paris 7, Robert Debré hospital (APHP), 48Bd, Sérurier, 75019 Paris, France. ${ }^{4}$ AFPA: Association Française de Pédiatrie Ambulatoire 4 rue Parmentier, F54270, Essey les Nancy, France. ${ }^{5}$ National Reference Center for Pneumococci, AP-HP, HEGP 20, rue, Leblanc, F75015 Paris, France.

\section{Authors' contributions}

RC designed the study and drafted the manuscript. CL coordinated the participating centers, was responsible for the statistical analysis and drafted the manuscript. EB helped draft the manuscript. FT and VD were the main investigators and helped draft the manuscript. MB designed the database and helped draft the manuscript. EB and EV performed bacteriological analyses and helped draft the manuscript. All authors read and approved the final manuscript.

\section{Authors' information}

Robert Cohen is a French pediatric infectious disease specialist, scientific director of a research institute on pediatric community acquired infections (ACTIV) and scientific director of a vaccine network for healthcare workers (Infovac-France). His main research interests are epidemiologic studies and clinical trials in community acquired infections, including pneumococcal diseases, rhinopharyngeal flora, and vaccines. He has published more than 45 papers in English.

\section{Competing interests}

The authors declare that they have no competing interests.

Received: 11 October 2011 Accepted: 7 March 2012

Published: 7 March 2012

\section{References}

1. Faden H, Stanievich J, Brodsky L, Bernstein J, Ogra PL: Changes in nasopharyngeal flora during otitis media of childhood. Pediatr Infect Dis $J$ 1990, 9(9):623-626.

2. Wertheim HF, Melles DC, Vos MC, van Leeuwen W, van Belkum A, Verbrugh HA, Nouwen $\mathrm{JL}$ : The role of nasal carriage in Staphylococcus aureu infections. Lancet Infect Dis 2005, 5(12):751-762. 
3. Cohen R, Levy C, Hentgen V, Boucherat M, De La-Rocque F, D'Athis P, Bingen E: Relationship between clinical signs and symptoms and nasopharyngeal flora in acute otitis media. Clin Microbiol Infect 2006, 12(7):679-682.

4. Faden H, Duffy L, Wasielewski R, Wolf J, Krystofik D, Tung Y: Relationship between nasopharyngeal colonization and the development of otitis media in children. Tonawanda/Williamsville Pediatrics J Infect Dis 1997, 175(6):1440-1445.

5. Leibovitz E, Jacobs MR, Dagan R: Haemophilus influenza: a significant pathogen in acute otitis media. Pediatr Infect Dis J 2004, 23(12):1142-1152.

6. Howie VM, Ploussard JH, Lester RL Jr: Otitis media: a clinical and bacteriological correlation. Pediatrics 1970, 45(1):29-35.

7. Broides A, Dagan R, Greenberg D, Givon-Lavi N, Leibovitz E: Acute otitis media caused by Moraxella catarrhalis: epidemiologic and clinical characteristics. Clin Infect Dis 2009, 49(11):1641-1647.

8. Anonymous: French vaccinal calendar. Bull Epidemiol Hebd 2006, 2930:212-224[http://www.invs.sante.fr/beh/].

9. Gaudelus J, Cohen R: Evolution of PCV7 coverage in 2006 to 2010: analysis of health records. Medecine et Enfance 2011, 31(4):187-190.

10. Klugman KP: Efficacy of pneumococcal conjugate vaccines and their effect on carriage and antimicrobial resistance. Lancet Infect Dis 2001, 1(2):85-91.

11. Huang $S S$, Hinrichsen VL, Stevenson AE, Rifas-Shiman SL, Kleinman $K$, Pelton SI, Lipsitch M, Hanage WP, Lee GM, Finkelstein JA: Continued impact of pneumococcal conjugate vaccine on carriage in young children. Pediatrics 2009, 124(1):e1-e11.

12. Cohen R, Levy C, Bonnet E, Grondin S, Desvignes V, Lecuyer A, Fritzell B, Varon E: Dynamic of pneumococcal nasopharyngeal carriage in children with acute otitis media following PCV7 introduction in France. Vaccine 2010, 28(37):6114-6121.

13. Paradise $J$ L: On classifying otitis media as suppurative or nonsuppurative, with a suggested clinical schema. J Pediatr 1987, 111(6 Pt 1):948-951.

14. Cohen R, Levy C, Boucherat M, Langue J, de La Rocque F: A multicenter, randomized, double-blind trial of 5 versus 10 days of antibiotic therapy for acute otitis media in young children. J Pediatr 1998, 133(5):634-639.

15. Cohen R, Levy C, Boucherat M, Langue J, Autret E, Gehanno P, de La Rocque F: Five vs. ten days of antibiotic therapy for acute otitis media in young children. Pediatr Infect Dis J 2000, 19(5):458-463.

16. Cohen R, De La-Rocque F, Boucherat M, et al: A Randomized trial cefpodoximeproxetil 5 days vs amoxicillin-clavulanate 8 days in children with acute otitis media. Med Mal Infect 1997, 27:596-602.

17. Cohen R, Levy C, Thollot F, de La Rocque F, Koskas M, Bonnet E, Fritzell B, Varon E: Pneumococcal conjugate vaccine does not influence Staphylococcus aureu carriage in young children with acute otitis media. Clin Infect Dis 2007, 45(12):1583-1587.

18. Cohen R, Levy C, Bingen E, Koskas M, Nave I, Varon E: Impact of 13-Valent Pneumococcal Conjugate Vaccine On Pneumococcal Nasopharyngeal Carriage in Children with Acute Otitis Media. Pediatr Infect Dis J 2012.

19. Cohen R, Levy C, Bonnet E, Thollot F, Boucherat M, Fritzell B, Derkx V, Bingen $E$, Varon E: Risk factors for serotype 19A carriage after introduction of 7-valent pneumococcal vaccination. BMC Infect Dis 2011 11:95.

20. Cohen R, Levy C, de La Rocque F, Gelbert N, Wollner A, Fritzell B, Bonnet E, Tetelboum R, Varon E: Impact of pneumococcal conjugate vaccine and of reduction of antibiotic use on nasopharyngeal carriage of nonsusceptible pneumococci in children with acute otitis media. Pediatr Infect Dis J 2006, 25(11):1001-1007.

21. Clinical and Laboratory Standards Institute: Performance standards for antimicrobial susceptibility testing: 18th informational supplement. CLSI document M100-S18. Wayne, Pa 2008.

22. Madhi SA, Adrian P, Kuwanda L, Cutland C, Albrich WC, Klugman KP: Longterm effect of pneumococcal conjugate vaccine on nasopharyngeal colonization by Streptococcus pneumoniae-and associated interactions with Staphylococcus aureus and Haemophilus influenzae colonization-in HIV-Infected and HIV-uninfected children. J Infect Dis 2007, 196(11):1662-1666.

23. van Gils EJ, Veenhoven RH, Rodenburg GD, Hak E, Sanders EA: Effect of 7valent pneumococcal conjugate vaccine on nasopharyngeal carriage with Haemophilus influenzae and Moraxella catarrhalis in a randomized controlled trial. Vaccine 2011, 29(44):7595-7598.
24. Casey JR, Pichichero ME: Changes in frequency and pathogens causing acute otitis media in 1995-2003. Pediatr Infect Dis J 2004, 23(9):824-828,

25. Levy C, Thollot F, Corrard F, Lecuyer A, Martin P, Boucherat M, Koskas M, Romain $\mathrm{O}$, Goldrey M, Hausdorff WP, et al: [Acute otitis media in ambulatory practice: Epidemiological and clinical characteristics after 7 valent pneumococcal conjugate vaccine (PCV7) implementation.]. Arch Pediatr 2011, 18(6):712-718.

26. Sabuncu E, David J, Bernede-Bauduin C, Pepin S, Leroy M, Boelle PY, Watier L, Guillemot D: Significant reduction of antibiotic use in the community after a nationwide campaign in France, 2002-2007. PLoS Med 2009, 6(6):e1000084.

27. Dabernat H, Seguy M, Faucon G, Delmas C: Epidemiology of Haemophilus influenza strains collected in 2004 in France and in vitro assessment of their susceptibility to antibiotics. Med Mal Infect 2007, 37(6):320-324.

\section{Pre-publication history}

The pre-publication history for this paper can be accessed here: http://www.biomedcentral.com/1471-2334/12/52/prepub

doi:10.1186/1471-2334-12-52

Cite this article as: Cohen et al:: Nasopharyngeal flora in children with acute otitis media before and after implementation of 7 valent pneumococcal conjugate vaccine in France. BMC Infectious Diseases 2012 12:52.

\section{Submit your next manuscript to BioMed Central and take full advantage of:}

- Convenient online submission

- Thorough peer review

- No space constraints or color figure charges

- Immediate publication on acceptance

- Inclusion in PubMed, CAS, Scopus and Google Scholar

- Research which is freely available for redistribution

Submit your manuscript at www.biomedcentral.com/submit
Ciomed Central 